\title{
Filter bank decomposition of seismic data with application to compression and denoising
}

\author{
Laurent C. Duval*, Institut Français du Pétrole, France; Tage Røsten, Statoil Research Centre, Norway
}

\section{Summary}

The use of discrete wavelet based analysis, feature extraction, denoising, and compression methods have led to extremely interesting developments in the field of seismic data processing. Notwithstanding, discrete wavelets belong to a wider class of filter banks. The use of more general filter banks allows the design of filter coefficients matching the signal's properties. Consequently, general filter banks bring forth the performance of discrete wavelet based seismic data processing techniques. In this paper, we discuss basics of general filter bank theory, and its applications to seismic data compression and denoising. We show that properly designed filter banks are able to outperform discrete wavelets in both instances.

\section{Introduction}

From a very simplistic point of view, seismic signals can be viewed as a combination of three types of components: pure geophysical or geological information, redundancy of this information, and noise or alterations arising from different kinds of sources. This combination results in the unformal equation

$$
\text { seismic signal }=\text { information }+ \text { redundancy }+ \text { noise } .
$$

Each and every task capable of separating information, decreasing redundancy, and suppressing noise is generally not performed by one single method, but oftenly involves many geophysical processing tools. Besides, many seismic applications, including analysis, denoising, processing, and data management (e.g., data access, visualization, and compression) oftenly provide a concentration of seismic information. By reducing the dynamic range of the seismic data in some way, it is possible to discard redundancy and uncoherent noise in the seismic data set. Discrete wavelets provide such a representation. They have successfully been applied to seismic data analysis (Foster et al., 1997), to seismic signal denoising (Miao and Cheadle, 1998), and to seismic data compression (Villasenor et al., 1996).

Using a discrete wavelet formalism, wavelet decomposition is based on a low-pass and a high-pass filter, employed in an iterative scheme. Depending on the scheme used, such an iteration leads for example to the discrete wavelet transform (DWT) or to the discrete wavelet packet transform (DWPT). A decomposition scheme involving several stages of filters and sampling operators (e.g., upsampling and downsampling), is generally called a filter bank (FB for short) system. But FB theory is more general than discrete wavelets by involving more than 2 filters and having several different iterative schemes.

Since discrete wavelet decomposition is based on only 2 filters, discrete wavelets suffer from lacks in filter design abilities, as shown for compression by Røsten et al. (1999) and Duval and Nguyen (1999). In order to demonstrate their powerfulness we focus in this paper on more general filter banks, and their ef-

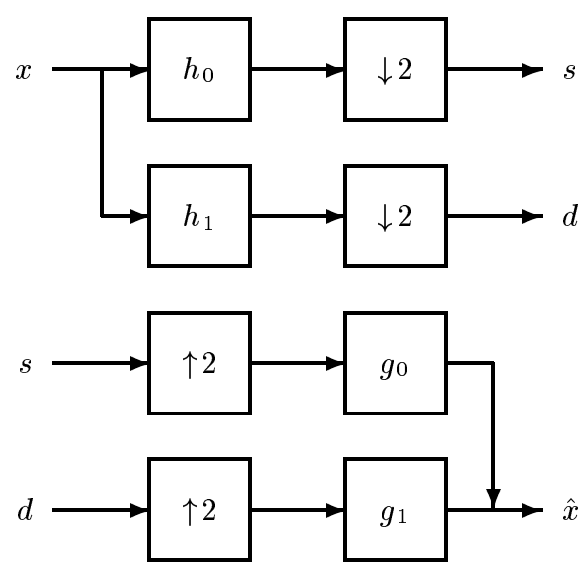

Fig. 1: 2-channel analysis and synthesis filter banks.

fectiveness for seismic data decomposition. The paper is organized as follows: first, we briefly recall some aspects of discrete wavelets, leading us to expose more general filter banks. Then, we describe how filter banks act on the data, and how FBs can be designed (application dependently) in order to match the properties of the seismic data. Advantages of general FBs over discrete wavelets are demonstrated for both data compression and denoising. The results exposed are obtained on actual marine field data: compression is applied to a 2-D common offset gather (COG) and denoising to a trace from the same COG.

\section{Discrete wavelets}

Fig. 1 shows a 2-channel filter bank system. At the analysis side, the input signal $x$ is filtered by a low-pass filter $h_{0}$ and a high-pass filter $h_{1}$ (i.e., 2-channel case). The outputs of the filters are maximally decimated or downsampled by 2 , i.e., every second sample is removed. The analysis FB splits $x$ into $s$, a coarse and smoother approximation (low-pass filtered version) of $x$, and into $d$, a detail signal (high-pass filtered version of $x$ ) from which the coarse approximation $s$ and the original signal $x$ differ. The resulting analysis decomposition is non-expansive: the number of coefficients after the analysis filtering is the same as the number of input samples due to the downsampling procedure. At the synthesis side, the $s$ and $d$ are upsampled by 2 (one zero is inserted between every second sample). Then the two upsampled signals are filtered by the corresponding low-pass and high-pass synthesis filters, denoted $g_{0}$ and $g_{1}$, respectively. The reconstructed signal $\hat{x}$ finally follows by a summation at the end. The signal $x$ can be perfectly reconstructed, i.e., $x=\hat{x}$, if the analysis and synthesis filters obey a so-called perfect reconstruction (PR) condition. That is, the synthesis FB cancels distortions introduced by the analysis FB. 


\section{Seismic data compression and denoising using filter banks}

The special case of DWT is based on a repeated low-pass analysis filtering of the approximation $s$, and results in a non-uniform filter bank with a so-called dyadic decomposition. In the 2-D case, one can apply a 1-level DWT decomposition first in the temporal direction and second in the spatial direction, and then iterate the DWT analysis filtering procedure on the lowpasslowpass subband (see Fig. 2 (a)). An alternative is to apply an $L$-level DWT decomposition first in time and second in space. The choice of the $h_{i}$ and $g_{i}$ filters is crucial. We refer to the book by Strang and Nguyen (1996) for a comprehensive survey of the construction of discrete wavelets. In the case of 2-channel (and $M$-channel) filter banks, we can divide the filters into three classes:

i) orthogonal PR filter banks: they generate an orthogonal basis for signal decomposition. $h_{1}, g_{0}$, and $g_{1}$ are retrieved from $h_{0}$;

ii) biorthogonal PR filter banks: they generate two bases where the $h_{i}$ 's are orthogonal to the $g_{i}$ 's, but the two bases are not mutually orthogonal. $g_{1}$ and $h_{1}$ are derived from $h_{0}$ and $g_{0}$, respectively;

iii) nonunitary filter banks.

Orthogonal bases, like the discrete Karhune-Loève transform, generally provide easier separation between signal and white noise components. For instance, Ioup and Ioup (1998) uses the orthogonal Daubechies Db20 wavelet for denoising, and shows its advantages over standard Fourier filtering. Nevertheless, the orthogonality constraint is a very strong condition in 2-channel FB design. For instance, it is not possible to have both orthogonal and symmetric (linear phase) filters except for trivial cases like for example the Haar wavelet. The orthogonality constraint is relaxed for biorthogonal and nonunitary filter banks. As a consequence, in the 2-channel case, they may simultaneously be PR and have linear phase - non-trivial - filter coefficients. The linear phase property is oftenly used to avoid phase distortion. Miao and Cheadle (1998) used the popular 9/7 biorthogonal linear phase wavelet in noise attenuation of seismic data. At the same time, many data compression applications involve the same 9/7 biorthogonal wavelet (e.g., Villasenor et al., 1996) or nonunitary filter banks (e.g., Røsten et al., 1999).

Some authors (see e.g., Vermeer et al., 1996), have pointed out that the DWT might not be the best suited decomposition scheme since it lacks accuracy in the high frequency subbands. Further-

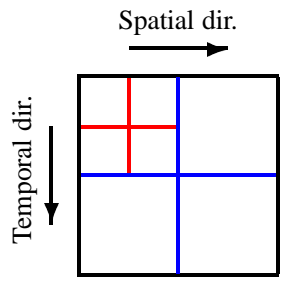

(a) Dyadic

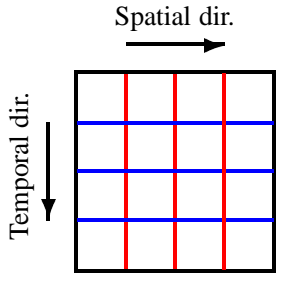

(b) Uniform

Fig. 2: 2-level dyadic (a) and 4-channel uniform (b) decomposition.

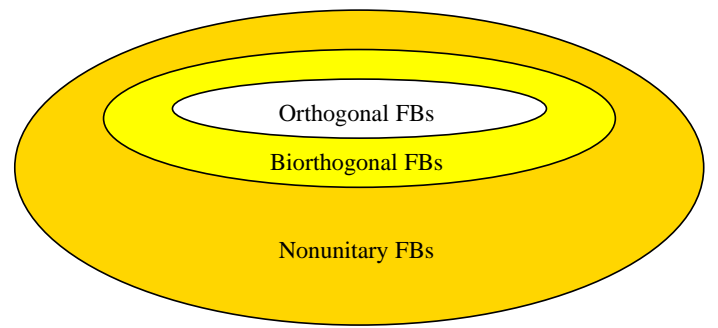

Fig. 3: Orth. and biorth. FBs are subsets of nonunitary FBs. Similarly, orth. and biorth. wavelets are subsets of ortho. and biorth. FBs.

more, a 2-channel FB intuitively possesses less degrees of freedom than FBs having three or more channels. Simultaneously orthogonality and linear phase constraints, in the $M$-channel case, $M>2$, are easier to meet since more filters add more coefficients to be tuned. By relaxing the orthogonality or biorthogonality constraint in the design of $M$-channel FBs, even greater degrees of freedom will arise (see Fig. 3).

\section{General filter banks}

Advances in digital signal processing have led to a more generic formalism for filter banks. A general structure of a maximally decimated $L+1$-channel analysis FB is given in Fig. 4 . The 1-D input signal $x$ is split into $L+1$ number of subbands by an analysis filter bank. The downsampling coefficients $\alpha_{i}$ are subject to the condition

$$
\sum_{i=0}^{L} 1 / \alpha_{i}=1,
$$

in order to keep the decomposition non-expensive. The special case $L=1$, i.e., a 2-channel FB with $\alpha_{0}=\alpha_{1}=2$, represents a 1-level DWT. An $L+1$-channel parallel analysis filter bank with $\alpha_{0}=2^{L}$ and

$$
\alpha_{i}=2^{L+1-i}, \quad 1 \leq i \leq L,
$$

has equivalent decomposition appearance to an $L$-level DWT. In the rest of the paper, we will mainly focus on uniform decompositions by general filter banks, setting all $\alpha_{i}$ 's to $M, M=L+1$.

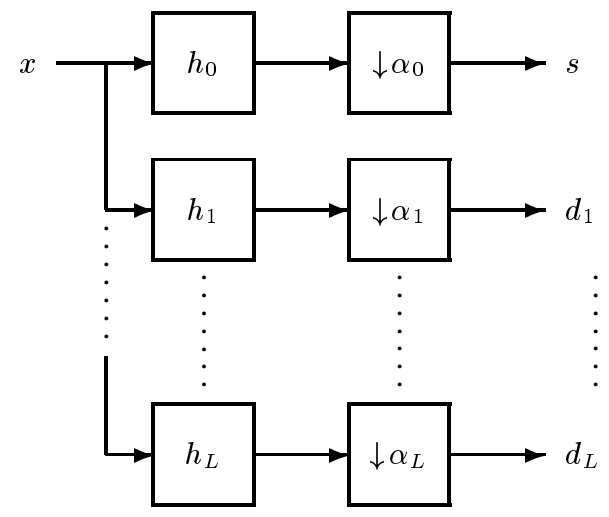

Fig. 4: An $L+1$-ch. analysis filter bank. 


\section{Seismic data compression and denoising using filter banks}

Fig. 2 (b) displays a uniform decomposition by utilizing a 4channel FB in two directions. The design and properties of filter banks depend on the targeted applications. An overview is given in the next section. We refer to Ramstad et al. (1995) for detailed issues on the design of general filter banks.

\section{Filter bank design}

The analysis filter bank is usually optimized to decorrelate the input signal, and this is obtained by maximizing the so-called coding gain. Additional practical constraints can be placed on the type of splitting and on length of the filters. For our purpose, we restrict to 8-channel 32-tap (each filter has 32 coefficients) linear phase FBs, i.e., $M=8$ and $K=32$. The 1-D coding gain $G$ of a uniform $M$-channel $K$-tap FB is in dB given by (see e.g., Røsten et al., 1999)

$$
G=10 \log _{10}\left[\prod_{i=0}^{M-1} A_{i}\right]^{-1 / M}
$$

provided that the synthesis filters have unit norm. The $A_{i}$ 's in equation (3) can be calculated from the analysis filters and the autocorrelation function (acf) $r_{x x}$ of the input signal:

$$
A_{i}=\sum_{j=0}^{K-1} \sum_{k=0}^{K-1} h_{i}(j) h_{i}(k) r_{x x}(j-k) .
$$

From equation (4) it is evident that we need to know the acf of the input signal to maximize equation (3). In the 2-D case, we have to investigate the sample to sample correlations both temporally and spatially. We choose a directional dependent separable statistical model with different zero-mean autoregressive (AR) processes to represent the acfs in the two directions.

For the particular case of common offset gathers, an AR(2) with $\rho_{1}=0.62$ and $\rho_{2}=0.10$ and an $\operatorname{AR}(1)$ with $\rho_{1}=0.70$ are utilized vertically and horizontally, respectively (Røsten et al., 1999). The coefficient $\rho$ with subscript 1 and 2 denotes normalized correlation coefficient at lag one and two.

The 8-channel 32-tap linear phase filter banks are optimized using suitable error terms for near PR, removal of blocking effects, high coding gain, and good stopband attenuation. Hence, a suitable error function $\varepsilon$ is

$$
\varepsilon=w_{P} \varepsilon_{P}+w_{B} \varepsilon_{B}+w_{G} \varepsilon_{G}+w_{S} \varepsilon_{S},
$$

where subscripts $P, B, G$, and $S$ denote PR, blocking effects, coding gain, and stopband attenuation, respectively. $\varepsilon_{G}$ is given by inverting equation 3. Full details can be found in (Ramstad et al., 1995; de Queiroz et al., 1996). The $w_{i}$ 's are proper weighting factors.

We optimize two different filter bank systems both in time (subscript $\mathrm{T}$ ) and space (subscript $\mathrm{S}$ ): $\mathrm{FB}-\mathrm{I}_{\mathrm{T}, \mathrm{S}}$ and $\mathrm{FB}-\mathrm{II}_{\mathrm{T}, \mathrm{S}}$. $\mathrm{FB}-\mathrm{I}$

\begin{tabular}{|l||cc|cc|}
\hline \hline Filter & FB-I & FB-II $_{\mathrm{T}}$ & FB-I & FB-II $_{\mathrm{S}}$ \\
\hline$G$ & 3.07 & 2.91 & 2.87 & 2.81 \\
\hline \hline
\end{tabular}

Table 1: Coding gains in $\mathrm{dB}$. is a nonunitary near-PR filter bank (Røsten et al., 1999) while FB-II is an orthogonal filter bank called GenLOT (Duval and Nguyen, 1999). For convenience, $w_{S}=0$ for FB-I since high stopband attenuation is more important for orthogonal denoising filter banks. Since FB-II is PR, $w_{P}=0$ for that filter bank system. For reference, Table 1 gives the resulting coding gain for the two optimized filter bank systems. Nonunitary FBs generally offer higher coding gains than orthogonal and biorthogonal FBs.

\section{Applications}

Depending on the application, additional stages are generally inserted between the analysis and the synthesis filter bank. I.e., an encoding block is utilized for seismic data compression, and a noise removal block is applied for denoising.

\section{Lossy seismic data compression}

In filter bank based lossy data compression, the subband coefficients are quantized, and finally encoded in an efficient way. Specifically, we apply the lossy data compression algorithm detailed in (Duval and Nguyen, 1999). This compression algorithm is based on an embedded coding scheme which progressively encodes and transmits the quantized and reordered subband coefficients by magnitude value. Three FBs are compared in the 2-D separable decomposition: the popular 9/7 biorthogonal wavelet (in both time and space), in addition to $\mathrm{FB}-\mathrm{I}_{\mathrm{T}, \mathrm{S}}$ and $\mathrm{FB}-\mathrm{II}_{\mathrm{T}, \mathrm{S}}$.

We compress a common offset gather containing 1101 number of shots with 870 time samples each. The compression ratio is ranging from $15: 1$ to $60: 1$. Fig. 5 shows that the coding gain optimized FBs, i.e., FB-I and FB-II, perform at least $3.5 \mathrm{~dB}$ better - in terms of signal-to-noise ratio (SNR) - than the 3-level 9/7 biorthogonal wavelet. FB-I and FB-II offer for all practical purposes the same results.

\section{White noise attenuation}

As a second filter bank example with relevance to seismic, we investigate a simple "hard" threshold method for denoising (Strang and Nguyen, 1996). A seismic trace from the common offset gather, or to be more correct the first 512 time samples of that trace, is corrupted with additive white Gaussian noise (AWGN) (see Fig. 6). After 1-D analysis filtering, subband coefficients below a certain threshold are set to zero. More sophisticated methods using "soft" or adaptive thresholding are under investigation.

The "denoised" seismic signal is reconstructed from the remaining subband coefficients through a corresponding 1-D synthesis filter. We use 4 different filter banks in this study: three of them based on the orthogonal Daubechies Dd20 wavelet with dyadic decomposition having 1, 3, and 5 number of levels, respectively. The last FB is the orthogonal and optimized $\mathrm{FB}-\mathrm{II}_{\mathrm{T}}$. We have compared the mean squared error (MSE) between the original and the denoised trace, using several thresholds, as shown in Fig. 7. We can see that the MSE level for FB-II $\mathrm{T}$ is, independently of the number of dyadic levels, almost always under those for the orthogonal Daubechies Db20 wavelet. That is, threshold- 


\section{Seismic data compression and denoising using filter banks}

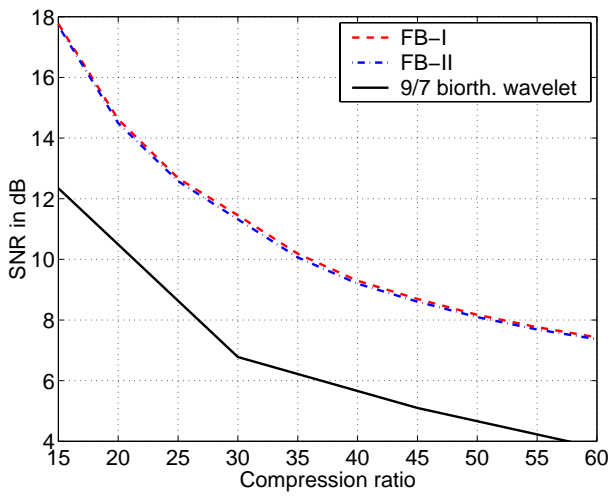

Fig. 5: SNR vs. compression ratio.
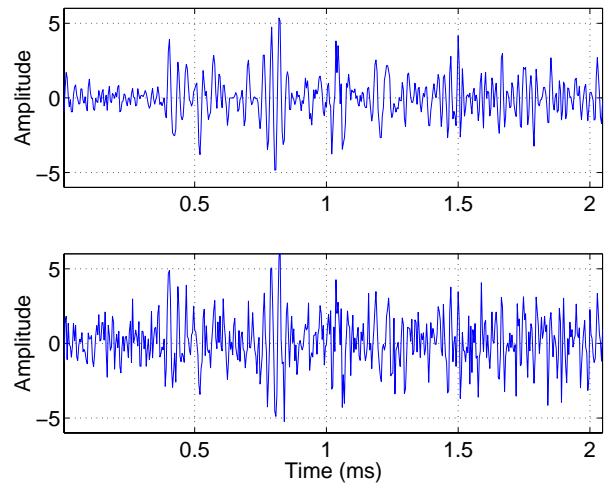

Fig. 6: Original data (top) and noisy data (bottom); MSE = 34.6.

ing the subband coefficients with $\mathrm{FB}-\mathrm{II}_{\mathrm{T}}$ results in an improvement of discrete wavelet based denoising. Furthermore, there is no need to choose a decomposition level, and denoising can easily be performed locally on subsets of 32 coefficients. A reasonable explanation for this better behavior is that the $\mathrm{FB}-\mathrm{II}_{\mathrm{T}}$ is designed to have good stopband attenuation, and thus matches the signal's frequency response better. Remark, we also can see that discarding more subband coefficients would result in more signal degradation: the MSE increases after the optimal threshold (obtained with the minimum MSE).

\section{Conclusions}

In this paper, we have demonstrated a flexible use of filter banks for both seismic data compression and noise attenuation. It is possible to design optimized filter banks application dependently: for instance, high coding gain helps to further decorrelate the seismic data and to concentrate the significant information of seismic data in a small number of coefficients, improving the results of seismic data compression. It is also possible to design the frequency responses of the analysis and synthesis filters to more efficiently separate signal from noise components. General filter banks are able to outperform discrete wavelets in both compression and noise attenuation. In addition, general filter banks have the feature of a more localized effect, thus overcoming some of the signal's unstationarities.

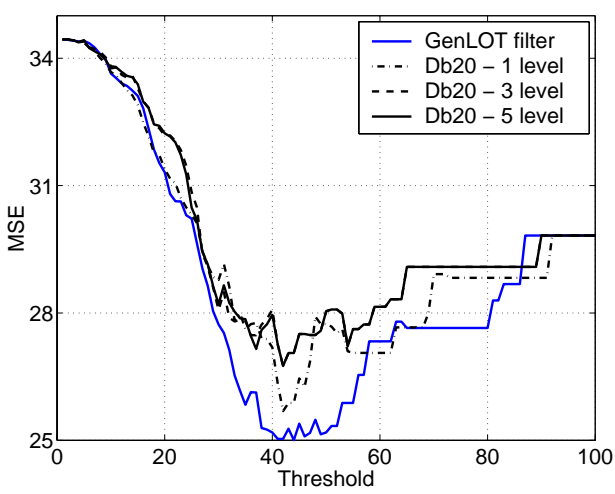

Fig. 7: Denoising with increasing thresholds. FB-II $\mathrm{T}_{\mathrm{T}}$ vs. Db20 using 1, 3 , and 5 dyadic decomposition levels.

\section{References}

de Queiroz, R. L., Nguyen, T. Q., and Rao, K. R., March 1996, The GenLOT: Generalized linear-phase Lapped Orthogonal Transform: IEEE Trans. on Signal Processing, 44, no. 3, 497507.

Duval, L. C., and Nguyen, T. Q., July 1999, Seismic data compression: a comparative study between GenLOT and wavelet compression: 44th SPIE Meeting, Proc. SPIE 3813, Wavelet Applications in Signal and Image Processing VII.

Foster, D. J., Lane, F. D., Mosher, C. C., and Wu, R.-S., 1997, Wavelet transforms for seismic data processing: 67th Ann. Internat. Mtg., Soc. Expl. Geophys., 1318-1321.

Ioup, J. W., and Ioup, G. E., 1998, Noise removal and compression using a wavelet transform: 68th Ann. Internat. Mtg., Soc. Expl. Geophys., 1076-1079.

Miao, X., and Cheadle, S., 1998, Noise attenuation with wavelet transforms: 68th Ann. Internat. Mtg., Soc. Expl. Geophys., 1072-1075.

Ramstad, T. A., Aase, S. O., and Husøy, J. H., 1995, Subband compression of images: principles and examples: Elsevier.

Røsten, T., Ramstad, T. A., and Amundsen, L., 1999, Seismic data compression, Part I: Subband coding of common-offset gathers: Submitted to Geophysics.

Strang, G., and Nguyen, T., 1996, Wavelets and filter banks: Wellesley-Cambridge Press.

Vermeer, P., Bragstad, H., and Orr, C., Nov. 1996, Aspects of seismic data compression: 66th Ann. Internat. Mtg., Soc. Expl. Geophys., 2031-2034.

Villasenor, J. D., Ergas, R. A., and Donoho, P. L., 1996, Seismic data compression using high-dimensional wavelet transforms: 66th Ann. Internat. Mtg., Soc. Expl. Geophys., 396405. 